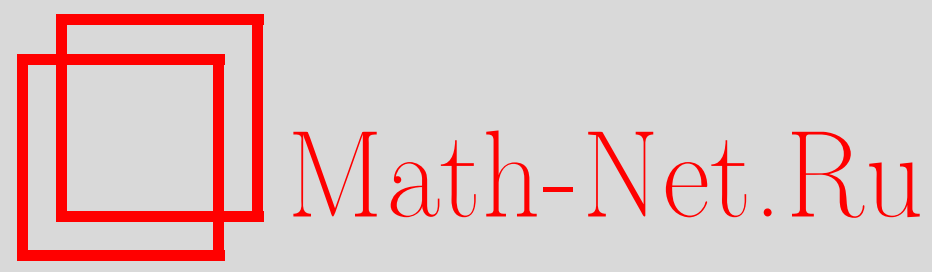

P. С. Исмагилов, Целочисленная группа Гейзенберга как бесконечная амальгама коммутативных групп, Матем. заметки, 2003, том 74, выпуск 5, 669-675

DOI: https://doi.org/10.4213/mzm299

Использование Общероссийского математического портала Math-Net.Ru подразумевает, что вы прочитали и согласны с пользовательским соглашением http://www.mathnet.ru/rus/agreement

Параметры загрузки:

IP : 35.173 .219 .149

26 апреля 2023 г., 03:11:41

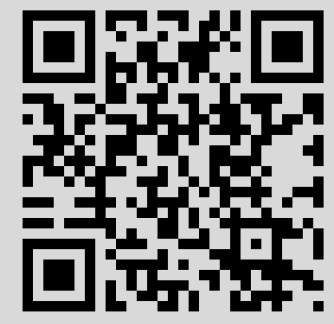




\section{ЦЕЛОЧИСЛЕННАЯ ГРУППА ГЕЙЗЕНБЕРГА КАК БЕСКОНЕЧНАЯ АМАЛЬГАМА КОММУТАТИВНЫХ ГРУПП}

\section{Р. С. Исмагилов}

Рассматривается $2 n$-мерная целочисленная решетка, $n>1$, со стандартной кососимметрической 2-формой, аддитивной по каждой переменной. Изучается семейство всех изотропных подрешеток. Доказывается, что амальгама этого семейства групп есть целочисленная группа Гейзенберга.

Библиографофия: 3 названия.

\section{1. Постановка задачи; описание результатов}

1.1. Амальгамы. Воспроизведем определение, данное, например, в статье Серpa [1]. Пусть дан набор груп $\left\{G_{\alpha}\right\}$, и для любых $\alpha, \beta$ дан набор гомоморфизмов $f_{\alpha \beta}$ : $G_{\alpha} \rightarrow G_{\beta}$ (этот набор может быть пустым для некоторых $\alpha, \beta$ ). Все это будем кратко записывать в виде $\left\{G_{\alpha}, f_{\alpha, \beta}\right\}$ и назьвать семейством (состоящим из групп и гомоморфизмов). Назовем представлением рассматриваемого семейства в групе Г такой набор гомоморфизмов $\Phi_{\alpha}: G_{\alpha} \rightarrow \Gamma$, что $\Phi_{\alpha}=\Phi_{\beta} \circ f_{\alpha \beta}$ для всех $\alpha, \beta$. Представление будем записьвать в виде $\Gamma,\left\{\Phi_{\alpha}\right\}$. Амальгама семейства $\left\{G_{\alpha}, f_{\alpha \beta}\right\}$ - это, по определению, такое представление $\Gamma^{\circ},\left\{\Phi_{\alpha}^{\circ}\right\}$, что множество $\cup \Phi_{\alpha}^{\circ}\left(G_{\alpha}\right)$ порождает группу $\Gamma^{\circ}$ и выполнено следующее свойство универсальности: для любого представления $\Gamma,\left\{\Phi_{\alpha}\right\}$ существует такой гомоморфизм $p: \Gamma^{\circ} \rightarrow \Gamma$, что $\Phi_{\alpha}=p \circ \Phi_{\alpha}^{\circ}$ для всех $\alpha$.

В литературе последнего времени, используя термин "амальгама", нередко предполагают, что рассматриваемое семейство групп конечно. Между тем, в предлагаемой работе возникнут и бесконечные семейства. По этой причине, имея бесконечное семейство групп (и гомоморфизмов между ними), мы будем применять термин “бесконечная амальгама". (Добавим, что в [1] используется также название "индуктивньй предел"; однако, это название нередко употребляется в ином смысле, и мы не будем его применять. Автор благодарит рецензента, указавшего на существующие ньне разночтения в терминологии в рассматриваемой области.)

Для дальнейшего важен следующий пример семейства $\left\{G_{\alpha}, f_{\alpha, \beta}\right\} .\left\{G_{\alpha}\right\}$ - это некоторьй набор подгрупп фиксированной группы $G$, замкнутьй относительно пересечений и такой, что множество $\cup G_{\alpha}$ порождает группу $G$. Гомоморфизм $f_{\alpha, \beta}$ определен только в том случае, если $G_{\alpha} \subset G_{\beta}$, и сводится к включению $G_{\alpha} \rightarrow G_{\beta}$. Такое семейство будем записьвать в виде $\left\{G_{\alpha}\right\}$ (опустив, для краткости, упоминание о включениях $f_{\alpha, \beta}$ ).

Работа выполнена при поддержке Российскаго фонда фундаментальных исследований, грант № 01-01-00490. 
Пусть $\Gamma^{\circ},\left\{\Phi_{\alpha}^{\circ}\right\}$ - амальгама (возможно, бесконечная) этого семейства. Группа $\Gamma^{\circ}$ задается образующими, состоящими из пар $(g, \alpha), g \in G_{\alpha}$, и соотношениями $(g, \alpha)=(g, \beta)$ при $G_{\alpha} \subset G_{\beta}, g \in G_{\alpha},\left(g_{1}, \alpha\right)\left(g_{2}, \alpha\right)=\left(g_{1} g_{2}, \alpha\right),(g, \alpha)^{-1}=\left(g^{-1}, \alpha\right)$ при $g_{1}, g_{2}, g \in G_{\alpha}$.

Согласно определению имеется такой эпиморфизм $p: \Gamma^{\circ} \rightarrow G$, что $p \circ \Phi_{\alpha}^{\circ}$ тождествен на $G_{\alpha}$ для каждого $\alpha$. Группа Ker $p$ дает те соотношения, которые следует добавить к уже описанным, чтобы получить в точности групп $G$ (мы воздержимся от более точных формулировок). Если в качестве $\left\{G_{\alpha}\right\}$ взять, скажем, набор всех абелевых подгрупп из $G$, то группы $\Gamma^{\circ}$ и $\operatorname{Ker} p$ естественно (“функториально”) зависят от $G$; обозначим эти функторы через $\mathscr{A}, \mathscr{A}^{\circ}$. Явное “вычисление” значений этих функторов на конкретной группе представляет, возможно, небезынтересную задачу (ниже будет рассмотрен простой пример такого рода).

1.2. Постановка задачи, результаты. Перейдем к описанию содержания предлагаемой работы. Зафиксируем целое число $n>1$ и рассмотрим обычную аддитивную группу $\mathbf{Z}^{2 n}$. Зафиксируем в ней стандартный базис $\left\{e_{k}, f_{k}, 1 \leqslant k \leqslant n\right\}$; таким образом, $\mathbf{Z}^{2 n}$ состоит из всех векторов-строк вида $a=\sum_{k=1}^{n}\left(x_{k} e_{k}+y_{k} f_{k}\right), x_{k}, y_{k} \in \mathbf{Z}$. Эту строку будем также записывать в виде $a=\left(x_{1}, \ldots, x_{n} ; y_{1}, \ldots, y_{n}\right)$. Пусть, далее, $\tau(a, b)$, $a, b \in \mathbf{Z}^{2 n},-$ аддитивная по каждой переменной целочисленная кососимметрическая форма такая, что $\tau\left(e_{i}, e_{j}\right)=\tau\left(f_{i}, f_{j}\right)=\tau\left(e_{i}, f_{k}\right)=0$ для всех $i \neq k, j, \tau\left(e_{k}, f_{k}\right)=1$. Подгруппу $V \subset \mathbf{Z}^{2 n}$ назовем изотропной, если $\tau(a, b)=0$ для всех $a, b \in V$. Набор всех изотропных подгрупп обозначим через $\operatorname{Is}\left(\mathbf{Z}^{2 n}\right)$. Наша задача - описать (бесконечную) амальгаму семейства $\operatorname{Is}\left(\mathbf{Z}^{2 n}\right)$. С этой целью рассмотрим целочисленную групу Гейзенберга $H^{2 n+1}(\mathbf{Z})$, определяемую как множество $\mathbf{Z}^{2 n} \times \mathbf{Z}$ с групповой операцией $(a, x)(b, y)=(a+b, x+y+\tau(a, b))$. Для любой подгруппы $V \in \operatorname{Is}\left(\mathbf{Z}^{2 n}\right)$ вложение

$$
V \rightarrow H^{2 n+1}(\mathbf{Z}), \quad a \mapsto(a, 0),
$$

есть, очевидно, гомоморфизм групп.

Tеорема 1. Пусть $n>1$. Тогда группа $H^{2 n+1}(\mathbf{Z})$ с гомоморфизмами (1) есть бесконечная амальгама семейства $\operatorname{Is}\left(\mathbf{Z}^{2 n}\right)$.

Мы предполагали, что $n>1$. Разумеется, можно построить (аналогичньпм образом) бесконечную амальгаму и при $n=1$. Однако она малоинтересна (сводится к свободному произведению счетного набора бесконечных циклических групш). Таким образом, ограничение $n>1$ весьма существенно.

Несколько слов о предыстории и мотивировках. В работах автора [2], [3] рассматривалась аналогичная задача с заменой групшы $\mathbf{Z}^{2 n}$ на аддитивную группу линейного пространства $\mathbf{R}^{2 n}$ и целочисленной группы $H^{2 n+1}(\mathbf{Z})$ - на обычную группу Гейзенберга (построенную по невырожденной кососимметрической 2-форме $\tau$ ). Эта задача возникла из модификации канонических коммутационных соотношений квантовой механики (в форме Вейля). (Впрочем, в работах [2], [3] рассматривались также линейные пространства над произвольным полем нечетной характеристики.) С точки зрения теории групп представляется естественным желание избавиться от структуры линейного пространства; это и привело к “целочисленной" модификации задачи, решенной в [2], [3]. Основная идея доказательства теоремы 1 та же, что в [2]; однако, осуществление этой идеи в "целочисленном" случае требует некоторых новых соображений (им посвящен раздел 2.1). 
СлЕдСТВИЕ. Бесконечная амальгама семейства всех абелевых подгрупп группь $H^{2 n+1}(\mathbf{Z})$ есть прямое произведение $H^{2 n+1}(\mathbf{Z}) \times \mathbf{Z}$, в которое кажсдая абелева подгруппа $A \subset H^{2 n+1}(\mathbf{Z})$ влохсена по правилу $(a, x) \mapsto(a, x, x)$, где $(a, x) \in A$.

Таким образом, это следствие дает значения упомянутых вьше функторов (связанных с бесконечньми амальгамами семейства всех абелевых подгрупп) на целочисленной группе Гейзенберга; результат состоит в том, что $\mathscr{A}\left(H^{2 n+1}(\mathbf{Z})\right)=H^{2 n+1}(\mathbf{Z}) \times \mathbf{Z}$, $\mathscr{A}^{\circ}\left(H^{2 n+1}(\mathbf{Z})\right)=\mathbf{Z}$.

Другие примеры выгисления амальгам содержатся в [3].

\section{2. Доказательства}

2.1. Некоторые свойства формы $\tau$ на группе $\mathrm{Z}^{2 n}$. В этом разделе будут сформулированы предложения 1,2 , дающие основное техническое средство для доказательства теоремы 1 ; доказательство этих предложений будет приведено в пп. 2.3, 2.4.

Рассмотрим группу $\mathbf{Z}^{2 n}$ с формой $\tau$, указанной в 1 . Элементы $a, b$ из $\mathbf{Z}^{2 n}$ назовем ортогональными (относительно $\tau$ ), если $\tau(a, b)=0$; в этом случае пишем $a \perp b$. Рассмотрим всевозможные упорядоченные пары $(a, b)$, где $a, b$ - векторы из $\mathbf{Z}^{2 n}$. Нам понадобится переход от такой пары к новой паре по следующему правилу: к одному из членов $a, b$ этой пары прибавляется вектор, ортогональный к обоим членам; будем записывать такой переход в виде $(a, b) \rightarrow\left(a^{\prime}, b^{\prime}\right)$. Итак, речь идет о переходах вида

$$
\begin{aligned}
& (a, b) \rightarrow\left(a^{\prime}, b^{\prime}\right), \quad a^{\prime}=a, \quad b-b^{\prime} \perp a, \quad b-b^{\prime} \perp b, \\
& (a, b) \rightarrow\left(a^{\prime}, b^{\prime}\right), \quad b^{\prime}=b, \quad a-a^{\prime} \perp a, \quad a-a^{\prime} \perp b .
\end{aligned}
$$

Назовем пары $(a, b),\left(a^{\prime}, b^{\prime}\right)$ әквивалентны.ми, если их можно связать цепочкой переходов

$$
(a, b)=\left(a_{1}, b_{1}\right) \rightarrow \cdots \rightarrow\left(a_{k}, b_{k}\right)=\left(a^{\prime}, b^{\prime}\right),
$$

каждьй из которых имеет вид (2a) либо (2b) (легко видеть, что таким образом действительно получается отношение эквивалентности); эквивалентность пар записьваем в виде $(a, b) \sim\left(a^{\prime}, b^{\prime}\right)$.

Ясно, что если $(a, b) \sim\left(a^{\prime}, b^{\prime}\right)$, то $\tau(a, b)=\tau\left(a^{\prime}, b^{\prime}\right)$. Следующее предложение показьвает, что верно и обратное; в этом предложении предполагается, что $n>1$, и рассматриваются пары векторов $(a, b),\left(a^{\prime}, b^{\prime}\right)$, взятых из $\mathbf{Z}^{2 n}$.

ПРЕДЛОЖЕНИЕ 1. Пусть $\tau(a, b)=\tau\left(a^{\prime}, b^{\prime}\right)$. Тогдa $(a, b) \sim\left(a^{\prime}, b^{\prime}\right)$.

Чтобы сформулировать следующее предложение, обозначим через $\operatorname{Sp}(n)$ группу всех обратимых матриц $g$ порядка $2 n \times 2 n$ с целыми элементами таких, что преобразование $a \mapsto a g, a \in \mathbf{Z}^{2 n}$ (умножение строки на матрицу) сохраняет форму $\tau$, т.е. $\tau(a, b)=$ $\tau(a g, b g)$ при $a, b \in \mathbf{Z}^{2 n}$. Таким образом, $\operatorname{Sp}(n)$ - это целочисленная симплектическая группа. В дальнейшем мы отождествляем матрицу $g \in \operatorname{Sp}(n)$ с соответствующим ей автоморфизмом групшы $\mathbf{Z}^{2 n}$. Ниже мы используем базис $\left\{e_{k}, f_{k}, 1 \leqslant k \leqslant n\right\}$, введенный в разделе 1 .

ПРЕДЛОЖЕНИЕ 2. 1) Для любого вектора $а \in \mathbf{Z}^{2 n}$ существует такая матрица $g \in \mathrm{Sp}(n)$, что $\mathrm{ag}=\mathrm{re}_{1} ;$ здесь $r$ - это наибольший общий делитель координат вектора $а$. 
2) Для любых векторов $a, b$ из $\mathbf{Z}^{2 n}$ существует такая матрица $g \in \operatorname{Sp}(n)$, что $a g=x e_{1}, b g=y e_{1}+z f_{1}+t e_{2}$ (для некоторых иелых $\left.x, y, z, t\right)$.

3) Если $\tau(a, b)=1$, то существует такая матрица $g \in \operatorname{Sp}(n)$, что аg $=e_{1}$, $b g=f_{1}$.

4) Если $a, b, c \in \mathbf{Z}^{2 n}, \tau(a, b)=1$, то существует такая матрича $g \in \operatorname{Sp}(n)$, что $a g=e_{1}, b g=f_{1}, c g=y e_{1}+z f_{1}+t e_{2}$, где $y, z, t$ иелье.

Предложение 2 вполне элементарно, но доказательство предложения 1 - достаточно длинное; нам удобно отложить доказательство этих предложений до п. 2.3, 2.4 этого раздела. В следующих двух разделах, опираясь на предложения 1, 2, мы докажем теорему 1 и ее следствие, сформулированное в разделе 1.

2.2. Доказательство теоремы 1 и ее следствия. Рассмотрим любое представление семейства $I s\left(\mathbf{Z}^{2 n}\right)$ в некоторой группе Г. Его можно отождествить с отображением $\Phi: \mathbf{Z}^{2 n} \rightarrow \Gamma$ таким, что его ограничение на любую изотропную подгруппу из $\mathbf{Z}^{2 n}$ есть гомоморфизм; это свойство можно записать так

$$
\Phi(a+b)=\Phi(a) \Phi(b) \quad \text { при } a \perp b, \quad \Phi(0)=e_{\Gamma} .
$$

(Через $e_{\Gamma}$ обозначен нейтральньй элемент группы $Г$.)

Для доказательства теоремы 1 достаточно построить такой гомоморфизм $\Phi_{0}$ : $H^{2 n+1}(\mathbf{Z}) \rightarrow \Gamma$, что

$$
\Phi_{0}(a, 0)=\Phi(a), \quad a \in \mathbf{Z}^{2 n} .
$$

С этой целью определим для любой пары $(a, b)$, составленной из элементов группы $\mathbf{Z}^{2 n}$, элемент из Г по формуле

$$
w(a, b)=\Phi(a+b) \Phi(-b) \Phi(-a) .
$$

ЛЕмма 1. 1) Существует (и однозначно определен) такой гомоморфизм групп $\theta: \mathbf{Z} \rightarrow \Gamma$, что $w(a, b)=\theta(\tau(a, b))$ для всех $a, b$ из $\mathbf{Z}^{2 n}$.

2) Указанный гомоморфизм $\theta$ удовлетворяет условию $\theta(k) \Phi(a)=\Phi(a) \theta(k)$ для всех $a \in \mathbf{Z}^{2 n}, k \in \mathbf{Z}$.

ДокАЗАтЕльство. Разобьем его на шаги.

Шаг 1. Из свойства (4) вытекает, что элемент (6) не меняется, если к одному из векторов $a, b$ прибавить вектор из $\mathbf{Z}^{2 n}$, ортогональный к ним обоим (напомним, что имеется в виду ортогональность относительно формы $\tau)$. Отсюда следует, что если $(a, b) \sim\left(a^{\prime}, b^{\prime}\right)$ (эквивалентность векторов была определена в п. 2.1), то $w(a, b)=w\left(a^{\prime}, b^{\prime}\right)$. Применяя предложение 1 , получаем отсюда, что $w(a, b)$ зависит только от величины $\tau(a, b)$, т.е. $w(a, b)=\theta(\tau(a, b))$ для некоторого отображения $\theta: \mathbf{Z} \rightarrow \Gamma$. Ясно, что $\theta(0)=e_{\Gamma}$, ибо $\Phi(0)=e_{\Gamma}$. Мы построили отображение $\theta$, указанное в первом утверждении леммы. Осталось проверить, что это отображение является гомоморфизмом и удовлетворяет условию 2) леммы.

Шаг 2. Перепишем равенство (6) в виде $\Phi(a+b)=w(a, b) \Phi(a) \Phi(b)$. Применим это равенство к $\Phi(a+b+c)$, представив выражение $a+b+c$ сначала в виде $a+(b+c)$, затем в виде $(a+b)+c$. После очевидных преобразований приходим к равенству

$$
w(a, b+c) \Phi(a) w(b, c) \Phi(-a)=w(a+b, c) w(a, b) .
$$


Зафиксируем вектор $a \in \mathbf{Z}^{2 n}$ и число $r \in \mathbf{Z}$. Тогда существуют такие векторы $b, c$ из $\mathbf{Z}^{2 n}$, что $b \perp a, c \perp a, \tau(b, c)=r$ (действительно, в силу предложения 2 достаточно проверить это для вектора $a=x e_{1}$, а для этого достаточно положить $\left.b=r e_{2}, c=f_{2}\right)$. Для таких векторов из (7) получаем (используя только что построенное отображение $\theta$ ) paвенство $\Phi(a) \theta(r) \Phi(-a)=\theta(r)$. Этим доказано, что отображение $\theta$ обладает свойством, указанным во втором утверждении леммы.

Шаг 3. Взяв целые числа $x, y$, применим равенство (7) к векторам $a=e_{1}, b=x f_{1}$, $c=y f_{1}$. Получим $\theta(x+y)=\theta(y) \theta(x)$. Напомним, что $\theta(0)=e_{\Gamma}$. Итак, $\theta-$ гомоморфизм групп, и лемма доказана.

Из этой леммы следует, что отображение $\Phi_{0}: H^{2 n+1}(\mathbf{Z}) \rightarrow \Gamma, \Phi_{0}(a, r)=\Phi(a) \theta(-r)$, есть гомоморфизм групп. Для него выполнено соотношение (5); этим доказана теоремa 1.

ДокАЖЕм следствие 1. Возьмем произвольноепредставление семейства всех абелевых подгрупп целочисленной групшы Гейзенберга $H^{2 n+1}(\mathbf{Z})$ в некоторой групе Г. Его можно отождествить с отображением $\Psi: H^{2 n+1}(\mathbf{Z}) \rightarrow \Gamma$ таким, что его ограничение на любую абелеву подгруппу есть гомоморфизм. Отображение $a \mapsto \Psi(a, 0), a \in \mathbf{Z}^{2 n}$, удовлетворяет условию (4); по теореме 1 существует такой гомоморфизм $p: H^{2 n+1}(\mathbf{Z}) \rightarrow \Gamma$, что $\Psi(a, 0)=p(a, 0)$ при $a \in \mathbf{Z}^{2 n}$. Положим $\Psi(0,1)=h_{0}, p(0,1)=h_{1}, h=h_{0}\left(h_{1}\right)^{-1}$. Тогда элементы $h_{0}, h_{1}, h$ перестановочны со всеми $\Psi(a, x),(a, x) \in H^{2 n+1}(\mathbf{Z})$, и $\Psi(a, x)=\Psi(a, 0) \Psi(0, x)=p(a, 0) h_{0}^{x}=p(a, x) h_{1}^{-x} h_{0}^{x}=p(a, x) h^{x}$. Итак, мы выразили отображение $\Psi: H^{2 n+1}(\mathbf{Z}) \rightarrow \Gamma$ через гомоморфизм $p: H^{2 n+1}(\mathbf{Z}) \rightarrow \Gamma$ по формуле $\Psi(a, x)=p(a, x) h^{x},(a, x) \in H^{2 n+1}(\mathbf{Z})$.

Группа $H^{2 n+1}(\mathbf{Z}) \times \mathbf{Z}$ состоит из элементов $(a, x, y)$ с правилом умножения $(a, x, y) \times$ $\left(a, x^{\prime}, y^{\prime}\right)=\left(a+a, x+x^{\prime}+\tau\left(a, a^{\prime}\right), y+y^{\prime}\right)$. Возьмем отображение $P: H^{2 n+1}(\mathbf{Z}) \times \mathbf{Z} \rightarrow \Gamma$, $P(a, x, y)=p(a, x) h^{y}$. Ясно, что это гомоморфизм; кроме того, $\Psi(a, x)=P(a, x, x)$. Этим доказано следствие 1.

2.3. Доказательство предложения 2. 1) Мы используем следующий простой факт: для любого ненулевого вектора $x=\left(x_{1}, \ldots, x_{m}\right) \in \mathbf{Z}^{m}$ существует такая матрица $g \in \mathrm{SL}(m, \mathbf{Z})$ (это группа матриц с целыми элементами и с определителем, равным 1$)$, что $x g=(r, 0, \ldots, 0)$, где $r$ есть наибольший обший делитель координат вектоpa $x$. Для доказательства первого утверждения предложения 1 возьмем любой вектор $a=\sum_{k=1}^{n}\left(x_{k} e_{k}+y_{k} f_{k}\right) \in \mathbf{Z}^{2 n}$. Для каждого $k=1, \ldots, n$ применим упомянутьй факт к двумерному подпространству с базисом $e_{k}, f_{k}$; умножив строку $\left(x_{k}, y_{k}\right)$ на подходящую матрицу, получаем строку $\left(r_{k}, 0\right)$. Таким образом, умножение строки $a$ на подходящую матрицу из $\mathrm{Sp}(n)$ приводит к строке $\sum_{k=1}^{n} r_{k} e_{k}$. Возьмем, далее, такую матрицу $h \in \mathrm{SL}(n, \mathbf{Z})$, что $\left(r_{1}, \ldots, r_{n}\right) h=(r, 0, \ldots, 0)$; через $h^{\prime}$ обозначим транспонированную матрицу. Тогда умножение на блочно-диагональную матрицу с блоками $h,\left(h^{\prime}\right)^{-1}$ (такое умножение сохраняет форму $\tau$ ) приводит данньй вектор к $r e_{1}$.

2) Возьмем теперь два целочисленных вектора $a, b$. В силу сказанного вьше можно считать, что $a=r e_{1}$. Применяя то же соображение к вектору $\sum_{k=2}^{n}\left(x_{k} e_{k}+y_{k} e_{k}\right)$, приходим к паре, указанной во втором утверждении предложения 2 .

3) Пусть $\tau(a, b)=1$. В силу сказанного данную пару можно привести (умножением на матрицу из $\operatorname{Sp}(n))$ к паре $\left(e_{1}, f_{1}+r_{1} e_{1}+r_{2} e_{2}\right)$. Применим преобразование $g$, переводящее векторы $f_{1}, f_{2}$ в $f_{1}-r_{1} e_{1}-r_{2} e_{2}$ и $f_{2}-r_{2} e_{1}$ (соответственно) и оставляющее на 
месте векторы $e_{1}, e_{2}, e_{i}, f_{i}, i>2$; легко видеть, что оно принадлежит группе $\operatorname{Sp}(n) . \mathrm{B}$ результате приходим к паре $e_{1}, f_{1}$. Этим доказано второе утверждение предложения 2 .

4) Пусть, наконец, даны векторы $a, b, c$, и $\tau(a, b)=1$. Согласно уже доказанному, существует такая матрица $g \in \operatorname{Sp}(n)$, что $a g=e_{1}, b g=f_{1}, c g=y e_{1}+z f_{1}+h$, и $h$ есть целочисленная линейная комбинация векторов $e_{i}, f_{i}, 2 \leqslant k \leqslant n$. Взяв такую матрицу $g \in \mathrm{Sp}(n-1)$, что $h g=t e_{2}$, приходим к последнему утверждению предложения 2.

2.4. Доказательство предложения 1. В следующей лемме собраны свойства отношения эквивалентности пар, введенного в п. 2.1.

Лемма 2. 1) Если $(a, b) \sim\left(a^{\prime}, b^{\prime}\right)$, mo $(x a, y b) \sim\left(x a^{\prime}, y b^{\prime}\right)$ для любых иельх $x, y$.

2) Если $\tau(a, b)=\tau\left(a^{\prime}, b^{\prime}\right)$ и векторы $a, b$ ортогональны (относительно формы $\tau$ ) каждому из векторов $a^{\prime}, b^{\prime}$, то $(a, b) \sim\left(a^{\prime}, b^{\prime}\right)$.

3) Любая пара $(a, b)$ әквивалентна некоторой паре вида $\left(r a^{\prime}, b^{\prime}\right)$, әде $r \in \mathbf{Z}$, $\tau\left(a^{\prime}, b^{\prime}\right)=1$.

ДокАЗАТЕЛЬСтво. 1). Пусть цепочка (3) соединяет эквивалентные пары $(a, b)$, $\left(a^{\prime}, b^{\prime}\right)$. Заменив каждую пару $\left(a_{i}, b_{i}\right)$ из цепочки $(3)$ парой $\left(x a_{i}, y b_{i}\right)$, получаем цепочку преобразований, переводящую пару $(x a, y b)$ в $\left(x a^{\prime}, y b^{\prime}\right)$.

2) Пусть пары $(a, b),\left(a^{\prime}, b^{\prime}\right)$ обладают свойством, описанным во втором утверждении леммы. Тогда цепочка преобразований $(a, b) \rightarrow\left(a+a^{\prime}, b\right) \rightarrow\left(a+a^{\prime}, b^{\prime}\right) \rightarrow\left(a^{\prime}, b^{\prime}\right)$ удовлетворяет условиям, определяющим понятие эквивалентности пар; этим доказано второе утверждение леммы.

3) Из предложения 2 следует, что третье утверждение леммы достаточно доказать для пары $\left(x e_{1}, y e_{1}+z f_{1}+t e_{2}\right)$. Но эта пара эквивалентна паре $\left(x e_{1}, y e_{1}+z f_{1}\right)$. Далее, дважды применяя второе (уже доказанное) утверждение леммы, получаем эквивалентности $\left(x e_{1}, y e_{1}+z f_{1}\right) \sim\left(x z e_{2}, f_{2}\right) \sim\left(x z e_{1}, f_{1}\right)$. Лемма доказана.

Из нее вытекает, что достаточно доказать предложение 1 для пар $(a, b),\left(a^{\prime}, b^{\prime}\right)$ таких, что $\tau(a, b)=\tau\left(a^{\prime}, b^{\prime}\right)=1$.

ЛЕмма 3. Если $\tau(a, b)=\tau\left(a, b^{\prime}\right)=1$, то $(a, b) \sim\left(a, b^{\prime}\right)$. (Аналогично, если $\tau(a, b)=\tau\left(a^{\prime}, b\right)=1, \operatorname{mo}(a, b) \sim\left(a^{\prime}, b\right)$.)

Другими словами, если к одному из векторов рассматриваемой пары прибавить вектор, ортогональный ко второму (но необязательно ортогональньй к обоим), то наша пара перейдет в эквивалентную.

ДокАЗАТЕльство. Используя предложение 2 (его последнее утверждение), можно считать, что речь идет о парах $\left(e_{1}, f_{1}\right),\left(e_{1}, y e_{1}+f_{1}+t e_{2}\right)$. Применяя определение эквивалентности векторов и второе утверждение леммы 2 , получаем $\left(e_{1}, y e_{1}+f_{1}+\right.$ $\left.t e_{2}\right) \sim\left(e_{1}, y e_{1}+f_{1}\right) \sim\left(e_{2}, f_{2}\right) \sim\left(e_{1}, f_{1}\right)$. Лемма доказана.

ЛЕмма 4. Если $\tau(a, b)=\tau\left(a^{\prime}, b^{\prime}\right)=1, \operatorname{mo}(a, b) \sim\left(a^{\prime}, b^{\prime}\right)$.

(Как было сказано после доказательства леммы 2 , из этой леммы следует предложение 1.)

ДокАЗАТЕльство. Пользуясь предложением 2, можно считать, что $(a, b)=\left(e_{1}, f_{1}\right)$, $\left(a^{\prime}, b^{\prime}\right)=\left(y e_{1}+z f_{1}+t e_{2}, u e_{1}+v f_{1}+w e_{2}+s f_{2}+h\right)$, где вектор $h \in \mathbf{Z}^{2 n}$ ортогонален векторам $e_{1}, f_{1}, e_{2}, f_{2}$; если $n=2$, то $h=0$. Согласно лемме 3 пара $\left(a^{\prime}, b^{\prime}\right)$ эквивалентна паре $\left(y e_{1}+z f_{1}+t e_{2}, u e_{1}+v f_{1}+s f_{2}\right.$ ) (ибо вектор $h+w e_{2}$ ортогонален первому 
вектору этой пары). Вектор $s e_{1}-v e_{2}$ ортогонален второму вектору последней пары; следовательно, по лемме 3 , прибавление к первому вектору пары вектора $k\left(s e_{1}-v e_{2}\right)$ ( $k$ целое) переводит пару в эквивалентную. При этом слагаемое $t e_{2}$ в первом векторе заменяется на $(t-k v) e_{2}$. Аналогичньп образом вектор $t f_{1}-y f_{2}$ ортогонален первому вектору рассматриваемой пары и, следовательно, прибавление ко второму вектору пары вектора $r\left(t f_{1}-y f_{2}\right)(r$ целое) переводит нашу пару в эквивалентную. При таком прибавлении слагаемое $v f_{1}$ заменится на $(v+r t) f_{1}$. Таким образом, коэффициенты $t$, $v$ меняются согласно правилам $t \rightarrow t-k v, v \rightarrow v+r t$. Ясно, что продолжая такие преобразования, мы можем обратить в нуль коэффициент $t$. Итак, пара $\left(a^{\prime}, b^{\prime}\right)$ эквивалентна паре $\left(y e_{1}+z f_{1}, u e_{1}+v f_{1}+s f_{2}\right) \sim\left(y e_{1}+z f_{1}, u e_{1}+v f_{1}\right)$ (здесь использована ортогональность вектора $f_{2}$ векторам $e_{1}, f_{1}$.) Далее, согласно второму утверждению леммы $2\left(y e_{1}+z f_{1}, u e_{1}+v f_{1}\right) \sim\left(e_{2}, f_{2}\right) \sim\left(e_{1}, f_{1}\right)$. Этим доказана лемма 4 и, тем самым, доказано предложение 1.

\section{СПИСОК ЦИТИРОВАННОЙ ЛИТЕРАТУРЫ}

[1] Серр ЖК. П. Деревья, амальгамы и $\mathrm{SL}_{2}$ // Математика. 1974. Т. 18. №1. С. 3-51.

[2] Исмагилов Р. С. Некоторые задачи об универсальных отображениях // Вестн. Тамбовского ун-та. Сер. Естеств. и тех. науки. 1997. Т. 2. № 4. С. 367-371.

[3] Исмагилов Р. С. Слабые $\mathrm{KKC} \mathrm{и} \mathrm{KAC}$ и индуктивные пределы семейств групп и алгебр // Функцион. анализ и его прилож. 2000. Т. 34. № 2. С. 75-78.

Московский государственный технический университет им. Н. Э. Баумана

Поступило 03.03 .2000

Исправленный вариант

27.05.2003 